\title{
El uso de las tecnologías de la información y la comunicación (TIC) en la prevención del juego patológico en población joven y adolescente
}

\author{
DANIEL CAMPOS BACAS \\ camposd@uji.es \\ SARA Nebot IBÁÑEZ \\ snebot@uji.es \\ SOlEDAD QUERO CASTELLANO \\ squero@uji.es \\ JUANA MARÍA BRETÓN-LÓPEZ \\ breton@uji.es
}

\section{Resumen}

Introducción. En España, la prevalencia del juego patológico (JP) oscila entre el 2 \% y el $3 \%$ de la población adulta (p. ej., Becoña, 1999) representando un problema relevante en jóvenes y adolescentes (Becoña, 2010). Por este motivo, es necesario dedicar esfuerzos en la prevención de este problema, sobre todo en esta población. En este sentido, las tecnologías de la información y la comunicación (TIC) pueden tener un papel fundamental a la hora de prevenir, evaluar e intervenir en el JP. El objetivo de este trabajo fue llevar a cabo una revisión sobre la utilización de las TIC en el ámbito de la prevención del JP en esta población. Método. Se realizó una búsqueda en bases de datos como Psyclnfo, ISI Web of Knowledge, Scopus y Springer Link. Resultados. Se encontraron 6 programas de prevención para el JP en población joven y adolescente, así como 4 estudios en Canadá que utilizaban TIC (vídeos y presentaciones interactivas) en intervenciones breves para la prevención del JP. Solo se encontró 1 estudio que utilizaba las TIC para llevar a cabo un programa de prevención del JP (It's Your Lucky Day). En España, no se encontraron estudios sobre prevención del JP y las TIC. Conclusiones. Los resultados muestran que la investigación en el uso de las TIC para la prevención del JP en jóvenes y adolescentes es escasa. El uso de estas herramientas se presenta como una línea de investigación prometedora debido a las ventajas que plantea para esta población específica ( $p$. ej., Heider y Massanari, 2010).

Palabras clave: juego patológico, TIC, prevención, jóvenes y adolescentes.

\section{Abstract}

Introduction. In Spain, the prevalence of Pathological Gambling (PG) is around $2 \%$ and $3 \%$ of adults (e.g., Becoña, 1999), representing a significant problem in youth and 
adolescents (Becoña, 2010). For this reason, it is necessary to devote efforts in preventing this problem, especially in this population. In this line, Information and Communication Technologies (ICTS) may have a key role in preventing, assessing and treating PG. The aim of this study was to conduct a review of the use of ICTS in the field of pathological gambling prevention among young and adolescent. Method. We searched in databases as Psyclnfo, ISI Web of Knowledge, Scopus y Springer Link. Results. Six prevention programs for PG among young and adolescent were found, as well as 4 studies in Canada that used ICTS (vídeos and interactive presentations) for brief interventions to prevent gambling. Only 1 study that used ICTS to apply a prevention program for PG (It's Your Lucky Day) was found. In Spain, no study about the prevention for PG and ICTS was found. Conclusion. The results show that the use of ICTS for PG prevention among youth and adolescents is scarce. The use of ICTS may be a promising line of research due to the advantages arising for this specific population (e.g., Heider \& Massanari, 2010).

Keywords: Pathological Gambling, ICTS, prevention, youth and adolescent.

\section{Introducción}

Según la última edición del Manual Diagnóstico y Estadístico de los trastornos mentales (DSM-IV-TR; APA, 2000), el juego patológico (JP) se clasifica como un trastorno del control de los impulsos no clasificado en otros apartados, a pesar de que los criterios especificados para el diagnóstico de este trastorno son similares a los de los trastornos por consumo de sustancias y otras adicciones. Principalmente, el JP se caracteriza por ser un comportamiento de juego desadaptativo, persistente y recurrente que interfiere y altera la vida personal, familiar o profesional (APA, 2000), teniendo que cumplir al menos cinco de los diez criterios indicados en la tabla 1. En ella también se refleja la propuesta de Fisher (1992) para ajustar los criterios en niños de entre 11 y 16 años (DSM-IV-J).

Tabla 1

Criterios diagnósticos del juego patológico en el DSM-IV-TR y DSM-IV-J

1. Preocupación por el juego.

2. Necesidad de jugar con cantidades crecientes de dinero para conseguir el grado de excitación deseado.

3. Fracaso repetido en el esfuerzo para controlar, interrumpir o detener el juego.

4. Inquietud o irritabilidad cuando intenta interrumpir o detener el juego.

5. El juego se utiliza como estrategia para escapar de los problemas.

6. Después de pérdidas se regresa para escapar de los problemas.

7. Se engaña a los miembros de la familia y otros para ocultar el grado de implicación con el juego. DSM-IV-J: Se engaña a los miembros de la familia o amigos para ocultar el grado de implicación en el juego. 
8. Se comenten actos ilegales para financiar el juego.

DSM-IV-J: Se cometen actos ilegales/antisociales, gastar dinero de la comida, robar en casa o fuera para jugar.

9. Se ha arriesgado o perdido relaciones interpersonales, trabajos, etc.

DSM-IV-J: Se riñe con la familia o con los amigos más próximos y se hace peligrar la educación por el juego.

10. Se confía en que los demás proporcionen dinero que alivie la desesperada situación financiara.

Adaptado de Arbinaga (2000)

Diferentes autores han insistido en la conveniencia de diferenciar distintos tipos de jugadores, aunque de momento, no existe acuerdo entre ellos y la evidencia experimental que los sustenta es mínima (Robert y Botella, 2008). Autores como González (2010) entienden el JP como un continuo de problemas relacionados con el juego de azar: desde el juego saludable al juego patológico, pasando por el juego de riesgo y el juego problemático. Desde esta perspectiva, el juego pasaría de ser un entretenimiento a una necesidad. Por su parte, Ochoa y Labrador (1994) proponen cuatro grupos de jugadores: los jugadores sociales, los jugadores profesionales, los jugadores problema y jugadores patológicos. El jugador social sería aquel que juega por placer, de modo esporádico y con control sobre el juego. El profesional también mantiene un control sobre el juego, estudiando las jugadas de modo que le permitan obtener beneficios. El jugador problema sería aquel jugador que, aunque todavía mantiene un cierto control sobre su conducta de juego sin graves problemas en su vida laboral y familiar, dedica gran cantidad de tiempo y dinero al juego, presentando un alto riesgo de convertirse en jugador patológico. Por último, el jugador patológico sería aquel que ha perdido el control sobre su conducta de juego, con un deterioro personal, familiar y social. En este sentido, diversos autores han tratado de estructurar el proceso a través del cual una persona pasa de ser jugador social a convertirse en jugador patológico (p. ej., Custer, 1984; Custer y Milt, 1985). Generalmente se trata de un proceso largo, que puede durar años y que suele comenzar en la adolescencia (Robert y Botella, 2008).

EI JP es un problema de salud importante reconocido tanto por la Asociación Americana de Psiquiatría (APA) como por la Organización Mundial de la Salud (OMS) (González, 2010). Los porcentajes de prevalencia varían del $1 \%$ al $2 \%$ en Estados Unidos (Shaffer, Hall y Van der Bilt, 1997), Canadá (Ladouceur, 1996) y Europa (Becoña, 1996). Los estudios han ido observando un incremento de este problema (p. ej., Arbindaga, 2000; Moreno, 2007), existiendo una preocupación generalizada sobre la elevada incidencia del JP en población de riesgo, como son los jóvenes y los adolescentes. En esta población se encuentran porcentajes entre el $1,7 \%$ y el $8 \%$ (Ladoucer y Mireault, 1988; Lesieur y Rosenthal, 1991). Más recientemente, en el panorama internacional se ha publicado nueva información sobre jóvenes, donde Westphal, Rush, Stevens y Johnson (2000) con una muestra de 11.736 estudiantes (11-18 años) reflejan que el $10,1 \%$ de los mismos indicaban problemas con el juego durante el año anterior y el $5,8 \%$ podía presentar JP. A su vez, datos de varios países indican que este problema se inicia en edades tempranas (Dell, Ruzicka y Palisi, 1981; Becoña, 2010).

En España, los estudios sobre menores de edad comenzaron a realizarse a partir del año 1996 los cuales se resumen en la tabla 2. 
Tabla 2

Estudios en España sobre el juego patológico en menores

\begin{tabular}{lccccc}
\hline Autor & Año & Muestra & Edad & J. Patológico & Contexto \\
\hline Arbinaga & 1996 & 105 & $14-17$ & $2,9 \%$ & Huelva \\
\hline Becoña, Gestal & 1996 & 1.200 & $12-14$ & $2,2 \%$ & Galicia \\
\hline $\begin{array}{l}\text { Villa, Becoña } \\
\text { Vázquez }\end{array}$ & 1997 & 2.185 & $11-16$ & $1,6 \%$ & Asturias \\
\hline Castro & 1999 & 153 & $15-17$ & $2,6 \%$ & I. Canarias \\
\hline
\end{tabular}

Tomado de Arbinaga (2000)

En los estudios que se recogen en la tabla 2 se encuentran porcentajes de prevalencia que oscilan entre el 1,6 \% y el 2,9\%. Estos datos muestran una alta prevalencia de JP en adolescentes. Datos más recientes indican que la prevalencia del JP en España se sitúa alrededor del $2 \%$ de la población adulta, representando un problema relevante en jóvenes y adolescentes (Becoña, 2010). La adolescencia, además, es un periodo de desarrollo muy importante para las conductas de juego teniendo en cuenta la alta prevalencia de conductas de riesgo en esta población (véase Echeburúa, Becoña y Labrador, 2010). Por último, tal y como recoge Becoña (1999), los jugadores patológicos predominan entre los más jóvenes: el 40 \% tiene entre 18 y 30 años.

Dado que, como muestran los datos, jóvenes y adolescentes tienen más probabilidades de desarrollar un problema de JP, es necesario dedicar esfuerzos en la prevención de este problema, sobre todo en esta población. En este sentido, las tecnologías de la información y la comunicación (TIC) pueden tener un papel fundamental a la hora de prevenir, evaluar e intervenir en el juego patológico.

La utilización de las TIC ha experimentando un incremento considerable en los últimos años demostrando su utilidad en el campo de la salud (Baños et al., 2012; Botella et al., 2009). En concreto, Internet ofrece un nuevo modo de dispensar tratamientos cognitivo-conductuales (TCC) (Andersson, 2009) y ha demostrado ser una intervención eficaz y de bajo coste para los trastornos de ansiedad y los trastornos del estado de ánimo (Botella, Hoffman y Moscovitch, 2004; Cuijpers y Schuurmans, 2007; Botella et al., 2010; Botella, Quero et al., 2008). Una ventaja fundamental de estos programas es que reducen el tiempo de contacto entre paciente y clínico, además de llegar a pacientes que no recibirían tratamiento de otro modo (Bauer, Golkaramnay y Kordy, 2005).

En población joven y adolescente las TIC presentan una serie de ventajas que pueden ayudar a fomentar la motivación para involucrarse en un programa de promoción de la salud. Entre las ventajas destacan que los jóvenes y adolescentes se identifican más con los avances tecnológicos, se sienten más animados a probarlos y no les frena su complejidad de uso. Asimismo, las TIC proporcionan un lenguaje atractivo y muy accesible (Internet) y permiten personalizar el tratamiento en función del perfil de la persona (Fox, 2000; Heider y Massanari, 2010).

Teniendo en cuenta las ventajas que presentan las TIC y los altos porcentajes de prevalencia de JP en población joven y adolescente, el objetivo principal de este estudio fue llevar a cabo una revisión sobre el uso de las TIC en el ámbito de la prevención del JP en población joven y adolescente. Además, se exploró el uso de las TIC en la prevención e intervención de otras adicciones, para comprobar si se habían utilizado y ver qué resultados se habían obtenido. 


\section{Método}

Se realizó una búsqueda en bases de datos como Psyclnfo, ISI Web of Knowledge, Scopus y Springer Link. En la tabla 3 se pueden ver los términos de búsqueda acotados. La revisión bibliográfica se llevó a cabo en dos fases. En una primera fase, la búsqueda se centró en revisar los programas de prevención de JP existentes en la literatura. En una segunda fase se realizó una búsqueda relacionada con el uso de las TIC en la prevención del JP, así como en la prevención e intervención de otras adicciones.

Tabla 3

Bases de datos y términos de búsqueda acotados en la búsqueda bibliográfica

\begin{tabular}{|c|c|}
\hline BASES DE DATOS & TÉRMINOS DE BÚSQUEDA \\
\hline & 1. ${ }^{a}$ Fase \\
\hline $\begin{array}{l}\text { Psyclnfo } \\
\text { ISI Web of Knowledge } \\
\text { Scopus }\end{array}$ & $\begin{array}{l}\text {-Prevention programs «and» pathological gambling } \\
\text {-Prevention approach «and» gambling } \\
\text {-Juego patológico «and» programas de prevención } \\
\text {-Prevention of gambling }\end{array}$ \\
\hline \multicolumn{2}{|l|}{ Springer Link } \\
\hline & $\begin{array}{l}\text { 2. }{ }^{a} \text { Fase } \\
\text {-ITCS «and» pathological gambling } \\
\text {-Computer prevention «and» pathological gambling } \\
\text {-Computer prevention «and» adictions } \\
\text {-Pathological gambling «and» a self applied Internet } \\
\text {-Pathological gambling «and» multimedia program }\end{array}$ \\
\hline
\end{tabular}

Se seleccionaron artículos que incluían programas de prevención del JP, estudios que utilizaron las TIC para la prevención e intervención del JP, así como estudios que usaban las TIC para la prevención e intervención de otras adicciones. La selección de los estudios se realizó siguiendo los criterios mencionados para explorar más ampliamente el estado del arte.

\section{Resultados}

Por lo que se refiere a programas de prevención del JP en jóvenes y adolescentes, se encontraron una variedad de programas de este tipo, sobre todo en el ámbito escolar. No obstante, pocos de ellos incluían datos acerca de su eficacia. En la tabla 4 se recogen los estudios encontrados que, además, mostraron evidencias de eficacia en la prevención del JP en esta población. 
Tabla 4

Programas de prevención para el juego patológico en jóvenes y adolescentes

\begin{tabular}{|c|c|c|c|c|}
\hline AUTORES & AÑO & POBLACIÓN & TIPO DE PROGRAMA & $\begin{array}{c}\text { PRINCIPALES } \\
\text { RESULTADOS DE EFICACIA }\end{array}$ \\
\hline $\begin{array}{l}\text { Gaboury } \\
\text { y Ladouceur }\end{array}$ & 1993 & $\begin{array}{l}11 \text { a } 18 \text { años } \\
(\mathrm{N}=289)\end{array}$ & $\begin{array}{l}\text { Educativo: } 3 \text { sesiones } \\
\text { Resumen del juego, discu- } \\
\text { siones de temas jurídicos, } \\
\text { manipulación de las posibi- } \\
\text { lidades de ganar, falacias } \\
\text { del juego, problemas con } \\
\text { el juego y habilidades de } \\
\text { afrontamiento. }\end{array}$ & $\begin{array}{l}\text {-Mejora del grupo interven- } \\
\text { ción en la educación sobre el } \\
\text { juego (a los } 6 \text { meses). } \\
\text {-Mejora en las habilidades de } \\
\text { afrontamiento tras el entrena- } \\
\text { miento, aunque no se mantie- } \\
\text { nen a los } 6 \text { meses. } \\
\text {-No se observan mejoras en } \\
\text { las conductas y actitudes de } \\
\text { juego }\end{array}$ \\
\hline
\end{tabular}

\begin{tabular}{|c|c|c|c|c|}
\hline Takushi et al. & 2004 & $\begin{array}{l}\text { Estudiantes en } \\
\text { riesgo de pro- } \\
\text { blemas con el } \\
\text { juego } \\
\text { (18-21 años) }\end{array}$ & $\begin{array}{l}\text { (BASICS) } 4 \text { componentes: } \\
\text { 1. Corrección de creencias } \\
\text { disfuncionales } \\
\text { 2. Solución de problemas } \\
\text { 3. HHSS } \\
\text { 4. Prevención de recaídas }\end{array}$ & $\begin{array}{l}\text { - Reducción en las conductas } \\
\text { de juego. } \\
\text { - Reducción en la frecuencia } \\
\text { de juego } \\
\text { - Reducción de los episodios } \\
\text { de alcohol y juego. }\end{array}$ \\
\hline $\begin{array}{l}\text { Ferland, } \\
\text { Ladouceur } \\
\text { y Vitaro }\end{array}$ & 2005 & $\begin{array}{l}\text { Estudiantes } \\
\text { (13 a } 18 \text { años) }\end{array}$ & $\begin{array}{l}\text { Educativo: } 3 \text { sesiones: } \\
\text { Naturaleza del juego, fala- } \\
\text { cias, juego excesivo y so- } \\
\text { lución de problemas. }\end{array}$ & $\begin{array}{l}\text { - Mejora a los } 3 \text { meses del } \\
\text { grupo intervención en los } \\
\text { conocimientos sobre el } \\
\text { juego y reducción de creen- } \\
\text { cias erróneas. }\end{array}$ \\
\hline $\begin{array}{l}\text { Turner, Mac- } \\
\text { donald, Bar- } \\
\text { toshuk y } \\
\text { Zangeneh }\end{array}$ & 2008 & $\begin{array}{l}\text { Estudiantes } \\
\text { (10 a } 18 \text { años) }\end{array}$ & $\begin{array}{l}\text { Programa de } 1 \mathrm{~h} \text { : } \\
\text { Información sobre el azar } \\
\text { y habilidades de afronta- } \\
\text { miento adaptativas (solu- } \\
\text { ción de problemas y apoyo } \\
\text { social). }\end{array}$ & $\begin{array}{l}\text { - Diferencias significativas } \\
\text { entre grupos en la adquisi- } \\
\text { ción de conocimientos: } \\
\left(\mathrm{F}(1,360)=6,8 ; p<0.01, \eta^{2}\right. \\
=0.02) \text {. } \\
\text { - Diferencias en la puntuación } \\
\text { de los test pre-post: } \\
F(14.4)=5.7, p<0.05 \text {. }\end{array}$ \\
\hline
\end{tabular}

\begin{tabular}{|c|c|c|c|c|}
\hline $\begin{array}{l}\text { Turner, } \\
\text { MacDonald } \\
\text { y Somerset }\end{array}$ & 2008 & $\begin{array}{l}\text { Estudiantes } \\
\text { (15 a } 18 \text { años) }\end{array}$ & $\begin{array}{l}\text { Currículum escolar } \\
\text { (Programa piloto). } \\
\text { Desarrollo del programa de } \\
1 \mathrm{~h} \text { de Turner et al. } 2008 .\end{array}$ & $\begin{array}{l}\text { Los resultados muestran dife- } \\
\text { rencias significativamente entre } \\
\text { grupos (experimental/control) } \\
F(1,198)=30.3 ; p<0,001 \text {. }\end{array}$ \\
\hline $\begin{array}{l}\text { Taylor } \\
\text { y Hillyard }\end{array}$ & 2009 & $\begin{array}{l}\text { Estudiantes } \\
\text { (12 años o } \\
\text { más) }\end{array}$ & $\begin{array}{l}\text { Sesión educativa sobre el } \\
\text { JP con lecturas, activida- } \\
\text { des y discusiones. }\end{array}$ & $\begin{array}{l}\text { Aumento en el conocimiento } \\
\text { del juego y de los efectos ne- } \\
\text { gativos que puede tener } \\
t(8,454)=-50,89 ; p=.000\end{array}$ \\
\hline $\begin{array}{l}\text { Williams, } \\
\text { Currie } \\
\text { y Wood }\end{array}$ & 2010 & $\begin{array}{l}\text { Estudiantes } \\
\text { (15 a } 18 \text { años) }\end{array}$ & $\begin{array}{l}\text { «Stacked Deck»: Progra- } \\
\text { ma educativo-escolar de } \\
5-6 \text { sesiones. }\end{array}$ & $\begin{array}{l}\text { Se observan cambios signifi- } \\
\text { cativos en las variables de- } \\
\text { pendientes desde el inicio al } \\
\text { seguimiento ( } 3-7 \text { meses) en } \\
\text { los tres grupos (control, inter- } \\
\text { vención, refuerzo). }\end{array}$ \\
\hline
\end{tabular}


Aunque no vamos a explicar de forma detallada los programas de prevención del JP resumidos en la tabla 4, cabe destacar aquí dos de estos estudios. Por una parte, el trabajo realizado en 1993 por Gaboury y Ladoucer, donde se llevó a cabo la evaluación de un programa de prevención de 3 sesiones educativas. Este trabajo representa uno de los primeros intentos en comprobar la eficacia de un programa preventivo para el JP en jóvenes y adolescentes (11 a 18 años), por lo que ha servido de referente para el desarrollo de posteriores programas preventivos de JP. Este es el caso del programa de prevención Stacked Deck (WiIliams, Currie y Wood, 2010), uno de los trabajos más reciente que ha demostrado la eficacia de un programa escolar canadiense de 5-6 sesiones para la prevención del JP. La sesión 1 se centra en la historia del juego y la ventaja de la casa, y se proporciona información sobre qué es el juego, la historia y los tipos, las probabilidades de los juegos de azar tradicionales y la insuperable ventaja a largo plazo de la casa. La sesión 2 trata los problemas con el juego y discute el potencial adictivo, los signos y síntomas, las causas y riesgos y dónde se puede recibir ayuda. En la sesión 3 se explican las Falacias del juego y se realizan ejercicios destinados a hacer más conscientes y menos susceptibles a los alumnos de los errores en el pensamiento y falacias del juego. La sesión 4 se dedica a la toma de decisiones y solución de problemas. En la sesión 5 se presentan las barreras en la toma de decisiones y solución de problemas. Por último, la sesión 6 (grupo refuerzo) se reserva para llevar a cabo un juego de preguntas con los alumnos.

En cuanto al uso de las TIC para la prevención del JP en jóvenes y adolescentes, se encontraron diversos estudios en Canadá. Así, el grupo de la Universidad de Laval (canadá) ha realizado varias evaluaciones de intervenciones cortas para la prevención de este problema en estudiantes de primaria (10 a 15 años). Entre estas destaca un vídeo educativo de 20 minutos (Ladouceur et al., 2004; Lavoie y Ladouceur 2004 y Ladouceur et al. 2005), una presentación interactiva de 40-60 minutos en la que se proporcionaba información sobre el juego y el juego patológico y se discutía con los estudiantes sobre aspectos relacionados con los juegos de azar como la ilusión de control y otras creencias erróneas (Ladouceur et al., 2003; Ladouceur et al., 2004), y la combinación de un vídeo educativo junto a una presentación interactiva de 40-60 minutos (Ferland, Ladouceur y Vitaro, 2002). En la misma línea, Wohl, Christie, Matheson y Anisman (2010) utilizaron un vídeo de animación educativo para la prevención del JP en participantes de 18 a 89 años de edad sin problemas con el juego patológico $(\mathrm{N}=242)$. El vídeo proporcionaba información sobre el funcionamiento de las máquinas tragaperras, la prudencia de establecer límites financieros y estrategias para evitar problemas. La herramienta utilizada en este estudio demostró tener un impacto positivo en relación a varias medidas importantes. Por ejemplo, se observó una disminución en el número de creencias erróneas, un aumento de creencias más certeras y un incremento en las estrategias de afrontamiento.

Uno de los trabajos más relevantes encontrados en la literatura revisada es el de Lemaire, De Lima y Patton (2004), en el que se presenta un programa piloto de prevención del JP para estudiantes aplicado a través de TIC (11-15 años). «It's Your Lucky Day», es un programa educativo y de sensibilización para el JP, divertido e interactivo. El objetivo de este programa canadiense era proporcionar información objetiva y fiable sobre el juego, a través de una sesión de presentación multimedia interactiva de 45-60 minutos y una plataforma web (www.luckyday. ca). En esta sesión se proporcionaba información y se discutía con los alumnos sobre los diferentes objetivos o temas del programa, como por ejemplo, la definición de los juego de azar, los mitos y las falacias de los juegos de azar, el funcionamiento de los juegos de azar, los signos de problemas con el juego y el conocimiento de centros de adicciones. El programa se completaba con un sitio web que recogía la información explicada en la sesión, así como un cuestionario interactivo para que los estudiantes pudieran poner a prueba sus conocimientos sobre los juegos de azar. Por otra parte, para comprobar la adquisición de los conocimientos, 
los participantes realizaron un cuestionario con diferentes ítems según los objetivos del programa. Se observaron diferencias significativas $(p<0,05)$ entre el grupo control y el experimental en la mayoría de los objetivos. Los resultados preliminares del programa mostraron un aumento del nivel de conocimiento de los alumnos en relación al juego y al juego patológico, así como una disminución de las falacias relacionadas con los juegos de azar. Este estudio representa uno de los primeros intentos formales en implantar un programa de prevención para el JP en población adolescente mediante el uso de las TIC, y concretamente con el apoyo de Internet.

En población clínica, se encontraron dos estudios, uno en Canadá y otro en España, donde se utilizaron las TIC para el tratamiento del JP en personas adultas. Carlbring y Smit (2008) presentaron una intervención de corte cognitivo-conductual de 8 semanas autoaplicada a través de Internet con apoyo semanal telefónico y por e-mail para el tratamiento de jugadores patológicos. Estos autores obtuvieron disminuciones en el JP, ansiedad y depresión, y un aumento en la calidad de vida tras el tratamiento en comparación a la condición control (lista de espera). Por su parte, Botella, Quero, Serrano, García-Palacios y Baños (2009) presentaron datos preliminares de una intervención para el JP mediante el uso de la Realidad Virtual en una serie de 3 casos. Los resultados pusieron de manifiesto la capacidad de los ambientes virtuales desarrollados por este grupo de investigación para activar el impulso a jugar en los 3 pacientes tratados. Además, se obtuvo una disminución importante tanto en el impulso por jugar como en el grado de creencia en los pensamientos irracionales después del tratamiento.

Respecto al uso de las TIC en los trastornos por consumo de sustancias y otras adicciones, una serie de estudios muestran la utilidad de estas tanto para la prevención como para la intervención. En las tablas 5 y 6 se recogen dichos estudios.

Tabla 5

Estudios que utilizan las TIC para la prevención de otras adicciones

\begin{tabular}{llll}
\hline AUTORES & AÑO & TIC & ADICCIÓN \\
\hline $\begin{array}{l}\text { Epstein y McGaha } \\
\text { Marsch et al. }\end{array}$ & 1999 & $\begin{array}{l}\text { Programa TV } \\
\text { Revisión de TIC en adiccio- } \\
\text { nes }\end{array}$ & $\begin{array}{l}\text { Sustancias } \\
\text { Sustancias }\end{array}$ \\
$\begin{array}{l}\text { Calleja, Pick, Reidl y Gonzá- } \\
\text { lez-Forteza, }\end{array}$ & 2010 & Juegos PC y workshop & Tabaco \\
$\begin{array}{l}\text { Rasal, Oliver, Vara, Agustí- } \\
\text { López y Baños }\end{array}$ & 2012 & Internet y juegos serios & $\begin{array}{l}\text { Sustancias (alcohol, ta- } \\
\text { baco, cocaína, canna- } \\
\text { bis, LSD, etc.) }\end{array}$ \\
\hline
\end{tabular}


Tabla 6

Estudios que utilizan las TIC para la intervención de otras adicciones

\begin{tabular}{llll}
\hline AUTORES & AÑO & TIC & ADICCIÓN \\
\hline Tossmann et al. & 2011 & Internet & Cannabis \\
Newman et al. & 2011 & $\begin{array}{l}\text { Revisión de tratamientos compu- } \\
\text { tarizados }\end{array}$ & Alcohol y tabaco \\
Gainsbury y Blaszczynski & 2011 & Internet & Revisión adicciones \\
Marsch & 2012 & Revisión trat. con TIC & Sustancias \\
\hline
\end{tabular}

Los estudios presentados en las tablas 5 y 6 sugieren que las TIC ya han mostrado utilidad en prevenir y tratar otras adicciones. Concretamente, en España, Rasal, Oliver, Vara, Agustí-López y Baños (2012) están evaluando la eficacia de un sistema que utiliza Internet y juegos serios para prevenir distintas adicciones en población infantil y adolescente.

\section{Conclusiones}

En la actualidad el JP puede considerarse como uno de los problemas de salud emergentes de mayor calado en nuestra sociedad (Sánchez-Hervás, 2003). Como se ha comentado anteriormente, los estudios de prevalencia muestran altos porcentajes de este problema en adultos y alarman sobre la alta incidencia del JP en población de riesgo, como son los jóvenes y los adolescentes (p. ej.: Arbindaga, 2000; Becoña, 2010). Además, se conoce que el JP se inicia en edades tempranas (Dell, Ruzicka y Palisi, 1981; Robert y Botella, 2008), sin olvidar que los jugadores patológicos predominan entre los más jóvenes: el $40 \%$ tiene entre 18 y 30 años (Becoña, 1999). En este contexto, se observa que los jóvenes y adolescentes tienen más probabilidades de desarrollar un problema con el juego, motivo por el cual la prevención del JP debería ser una de las prioridades a tener en cuenta.

En este sentido, los estudios revisados en este trabajo indican que el uso de las TIC en la prevención del JP se presenta como una línea de investigación prometedora, debido a las ventajas que plantea en esta población específica. El uso de estas herramientas favorece la implicación de los jóvenes y adolescentes en programas de promoción de la salud (Fox, 2000; Heider y Massanari, 2010). Sin embargo, la revisión llevada a cabo en este estudio también muestra que la investigación en el uso de las TIC para la prevención de este problema en jóvenes y adolescentes es escasa. Entre los estudios existentes, destacan diferentes experiencias en Canadá donde se han utilizado las TIC para la prevención del JP en jóvenes y adolescentes con resultados prometedores. It's Your Lucky Day es uno de los primeros estudios que utiliza las TIC (una presentación multimedia interactiva con el complemento de una página web) para establecer un programa de prevención del JP en estudiantes (11-15 años) de forma controlada. Sin embargo, en España, no se han encontrado hasta la fecha estudios que utilicen las TIC para la prevención de este problema. Este hecho, junto con el alto riesgo que presenta esta población a desarrollar un problema como el JP, el cual puede acarrear consecuencias muy negativas para la vida de la persona que lo padece (p. ej., pérdidas de dinero o endeudamientos, problemas familiares y de relaciones sociales, trastornos mentales asociados como depresión, ansiedad u otras adicciones) nos debe hacer reflexionar sobre la nece- 
sidad de dedicar esfuerzos y recursos en abrir líneas de investigación en el campo de la prevención de este problema.

\section{Referencias bibliográficas}

American Psychiatric Association (APA). (2000). Diagnostic and statistical manual of mental disorder (4. ${ }^{a}$ ed.) (DSM-IV-TR). Washington, DC: APA.

Andersson, G. (2009). Using the Internet to provide cognitive behaviour therapy. Behaviour Research and Therapy, 47, 175-180.

Arbinaga, F. (2000). Estudio descriptivo sobre el juego patológico en estudiantes (8-17 años): Características sociodemográficas, consumo de drogas y depresión. Adicciones. 12, n. $^{\circ} 4$, 493-505.

Baños, R. M., Guillén, V., García-Palacios, A., Quero, S. \& Botella, C. (2012). Las nuevas tecnologías en el tratamiento de los trastornos de ansiedad. Información Psicológica, 102, 28-46.

Bauer, S., Golkaramnay, V. \& Kordy, H. (2005). E-Mental-Health: Neue Medien in der psychosozialen Versorgung. Psychotherapeut, 50, 7-15.

Becoña, E. (1996). Prevalence surveys of problem and pathological gambling in Europe:The cases of Germany, Holland, and Spain. Journal of Gambling Studies, 12, 197-192.

Becoña, E. (1999). Bases teóricas que sustentan los programas de prevención de Drogas. Madrid: Plan Nacional sobre Drogas.

Becoña, E. (2010). Epidemiología del juego patológico. En Echeburúa, E., Becoña, E., Labrador, J. F. Fundación GAUDIUM (coords.) (2010), El juego patológico: Avances en la clínica y en el tratamiento. Madrid: Pirámide.

Blinn-Pike, L., Lokken, S. \& Jonkman, J. N. (2010). Adolescent Gambling: A Review of an Emerging Field of Research. Journal of Adolescent Health, 47, 223-236.

Botella, C., Gallego, M. J., García-Palacios, A., Guillen, V., Baños, R. M., Quero, S. \& Alcañiz, M. (2010). An Internet-Based Self-Help Treatment for Fear of Public Speaking: A Controlled Trial. Cyberpsychology Behavior, and Social Networking, 13, 407-421.

Botella, C., Hofmann, S. G. \& Moscovitz, D. A. (2004). A self-applied Internet-based intervention for fear of public speaking. Journal of Clinical Psychology In Session, 60: 1-10.

Botella, C., Quero, S., Baños, R. M., García-Palacios, A., Bretón-López, J., Alcañiz, M. \& Fabregat, S. (2008). Telepsychology and Self-Help: The treatment of phobias using the Internet. CyberPsychology \& Behavior, 11 (6), 659-664.

Botella, C., Quero, S., Serrano, B, García-Palacios, A. \& Baños, R. M. (2009). El uso de la Realidad Virtual en el tratamiento del Juego patológico. En J. Del Pozo, L. Perez \& Ferreras, M. (ed.), Adicciones y Nuevas tecnologías de la información y de la Comunicación. Perspectivas de su uso para la prevención y el tratamiento. Gobierno de La Rioja. Consejería de Salud.

Botella, C., Quero, S., Serrano, B., Baños, R. M. \& García-Palacios, A. (2009). Avances en los tratamientos psicológicos: La utilización de las Nuevas tecnologías de la Información y la Comunicación. Anuario de Psicología, 40 (2), 155-170.

Calleja, N., Pick, S., Reidl, L. \& Gonzalez-Forteza, C. (2010). Smoking prevention programs for girls. Salud mental, 33 (5), 419-427.

Carlbring, P. \& Smit, F. (2008). Randomized trial of internet-delivered self-help with telephone support for pathological gamblers. Journal of Consulting and Clinical Psychology, 76, 10901094.

Cuijpers, P. \& Schuurmans, J. (2007). Self-help interventions for anxiety disorders: An overview. Current Psychiatry Reports, 9, 284-290. 
Custer, R. \& Milt, H. (1985). When luck runs out. Help for compulsive gamblers and their families. Nueva York: Facts on File Publications.

Custer, R. L. (1984). Profile of the pathological gambler. Journal of Clinical Psychiatry, 45, 35-38.

Dell, L. J., Ruzicka, M. F. \& Palisi, A. T. (1981). Personality and other factors associated with the gambling addiction. International Journal of the Addictions, 16, 149-156.

Dickson, L. M., Derevensky, J. L. \& Gupta, R. (2002). The prevention of gambling problems in youth: A conceptual framework. J Gamb Stud, 18, 97-159.

Echeburúa, E., Becoña, E., Labrador, J. F. \& Fundación GAUDIUM (coords.) (2010), El juego patológico: Avances en la clínica y en el tratamiento. Madrid: Pirámide.

Epstein, J. \& McGaha, A. C. (1999). ATOD-TV: Evaluation of a multimedia program designed to educate the public about substance abuse. Computers in Human Behaviour, 15, 73-83.

Ferland, F., Ladouceur, R. \& Vitaro, F. (2005). Efficiency of a gambling prevention program for youths: Results from the pilot study. Encephale, 31(4), 427-436.

Ferland, F., Ladouceur, R. \& Vitaro, F. (2002). Prevention of problem gambling: Modifying misconceptions and increasing knowledge. Journal of Gambling Studies, 18(1), 19-29.

Fisher, S. E. (1992). Measuring pathological gambling in children: the case offruit machines in the U. K. Journal of Gambling Studies, 8, 263-285.

Fox, S. (2000). The online health care revolution: How the web helps Americans take better care of themselves. New York: The Pew internet and American life project.

Gaboury, A. \& Ladouceur, R. (1993). Evaluation of a prevention program for pathological gambling among adolescents. J Prim Prev;14: 21-28.

Gainsbury, S. \& Blaszczynski, A. (2011). A systematic review of Internet-based therapy for the treatment of addictions. Clinical Psychology Review, 31, 490-498.

González, A. (2010). Juego patológico: concepto, comorbilidad y tipologías. En Echeburúa, E., Becoña, E., Labrador, J. F., Fundación GAUDIUM (coords.) (2010), El juego patológico: Avances en la clínica y en el tratamiento. Madrid: Pirámide

Heider, D. \& Massanari, A. L. (2010). Friendship, closeness, and disclosure in Second Life. International Journal of Gaming and Computer-Mediated Simulations, 2(3). 61-74.

Irurita, I. M. (1996). Estudio sobre la prevalencia de los jugadores de azar en Andalucía. Sevilla. Comisionado para la Droga, Consejería de Asuntos Sociales, Junta de Andalucía.

Ladoucer, R. (1996). The prevalence of pathological gambling in Canada. Journal of Gambling Studies, 12, 129-142.

Ladouceur, R. \& Mireault, C. (1988). Gambling behaviors among high school students in the Quebec area. J Gamb Behav; 4: 3-12.

Ladouceur, R., Ferland, F. \& Vitaro, F. (2004b). Prevention of problem gambling: Modifying misconceptions and increasing knowledge among Canadian youths. Journal of Primary Prevention, 25(3), 329-335.

Ladouceur, R., Ferland, F., Roy, C., Pelletier, O., Bussières, E.-L. \& Auclair, E. (2004a). Prévention du jeu excessif chez les adolescents: Une approche cognitive. Journal de Thérapie Comportementale et Cognitive, 14(3), 124-130.

Ladouceur, R., Ferland, F., Vitaro, F. \& Pelletier, O. (2005). Modifying youths' perception toward pathological gamblers. Addictive Behaviors, 30, 351-354.

Lavoie, M.-P. \& Ladouceur, R. (2004). Prevention of gambling among youth: Increasing knowledge and modifying attitudes toward gambling. Journal of Gambling Issues (formerly eGambling: The Electronic Journal of Gambling Issues), 10.

Lemaire, J., De Lima, S. \& Patton, D. (2004). It's Your Lucky Day: Program evaluation. Winnipeg, Canada: The Addictions Foundation of Manitoba. 
Lesieur, H. R. \& Rosenthal, R. J. (1991). Pathological gambling: A review of the literatura (prepared for the American Psychiatric Association Task Force on DSM-IV Committee on Disorders okf Impulse Control Not Elsewhere Classified). Journal of Gambling Studies, 7(1), 5-39.

Marsch, L. A. (2012). Leveraging Technology to Enhance Addiction Treatment and Recovery. Journal of Addictive Diseases, 31 (3), 313-318.

Marsch, L. A., Bickel W. K. \& Grabinski, M. J. (2007). Application of interactive, computer technology to adolescent substance abuse prevention and treatment. Adolesc Med State Art Rev, 18(2), 342-356.

Mira, A., Molinari, G., García-Palacios, A., Navarro, M. V., Riera López del Amo, A. Pérez-Ara, M. Á., Agustí-López, A. I. \& Botella, C. (2012). La utilización de las Tecnologías de la Información y la Comunicación (Tic) en el ámbito de los tratamientos psicológicos. Fòrum de recerca Universitat Jaume I, 16, 1071-1083.

Moreno, S. (coord.) (2007). Guía clínica. Actuar ante el juego patológico. Sevilla: Consejería para la Igualdad y Bienestar Social de la Junta de Andalucía.

Newman, M. G., Szkodny, L. E., Llera, S. J. \& Przeworski, A. (2011). A review of technologyassisted self-help and minimal contact therapies for drug and alcohol abuse and smoking addiction: Is human contact necessary for therapeutic efficacy? Clinical Psychology Review, 31, 178-186.

Ochoa, E. \& Labrador, F. J. (con la colaboración de E. Echeburúa, E. Becoña \& M. A. Vallejo) (1994). El juego patológíco. Barcelona: Plaza \& Janés.

Quero, S., Rasal, P., Nebot, S., Bretón-López, J. \& Botella, C. (2012). El uso de TICs para el tratamiento de la fobia a animales pequeños. Ponencia presentada en el VIII Congreso Nacional de la Asociación Española de Psicología Clínica y Psicopatología, Almería, España.

Rasal, P., Oliver, E., Vara, L., Agustí-López, A. I. \& Baños, R. M. (2012). Preven-tic Formativo: programa on-line para la prevención del consumo de drogas dirigido a niños y adolescentes. VIII Congreso Nacional de la Asociación Española de Psicología Clínica y Psicopatología. Octubre. Almería.

Robert, C. \& Botella, C. (2008). Trastornos de control de impulsos: el juego patológico. En Belloch, Sandín y Ramos (eds.), Manual de psicopatología (424-448).

Sanchez-Hervás, E. (2003). Juego patológico: un trastorno emergente. Trastornos Adictivos, 5(2):75-87.

Shaffer, H. Hall, M. N. \& Van der Bilt, J. (1997). Estimating prevalence of disordered gambling behavior in the United States and Canada: A meta-analysis. Boston: Havard Medical Division on Addictions.

Takushi, R. Y., Neighbors, C., Larimer, M. E., Lostutter, Ty. W., Cronce, J. M. \& Marlatt, G. A. (2004). Indicated Prevention of Problem Gambling Among College Students. Journal of Gambling Studies, 20 (1).

Taylor, L. M. \& Hillyard, P. (2009). Gambling awareness for youth: An analysis of the “Don't Gamble Away our Future" program. International Journal of Mental Health and Addiction, 7(1) 250-261.

Tejeiro, R. (1996). Estudio sobre el juego de azar en Algeciras. Prevalencia y características sociodemográficas. Algeciras. JARCA.

Tossman, H.-P., Jonas, B., Tensil, M.-D., Lang, P. \& Struber, E. (2011). A Controlled Trial of an Internet-Based Intervention Program for Cannabis Users. Cyberpsychology, Behaviour and Social Networking, 14 (11), 673-679. 
Turner, N. E., Macdonald, J. \& Somerset, M. (2008). Life Skills, Mathematical Reasoning and Critical Thinking: A Curriculum for the Prevention of Problem Gambling. J Gamb/ Stud, 24: 367-380.

Turner, N. E., Macdonald, J., Bartoshuk, M. \& Zangeneh, M. (2008). The Evaluation of a 1-h Prevention Program for Problem Gambling. Int J Ment Health Addiction, 6: 238-243.

Westphal, J. R., Rush, J. A, Stevens, L. \& Johnson, L. (2000). Gambling behavior of Louisiana students in grades 6 through 12. Psychiatric Services. 51, 1, 96-99.

Wohl, M. J. A., Christie, K., Matheson, K. \& Anisman, H. (2010). Animation-Based Education as a Gambling Prevention Tool: Correcting Erroneous Cognitions and Reducing the Frequency of Exceeding Limits Among Slots Players. J Gamb/ Stud, 26: 469-486. 